\title{
EDITORIAL
}

\section{Anxiety disorders and systems biology research: a challenging brave new world}

\author{
Jeffrey P. Kahn, ${ }^{1}$ (iD Antonio E. Nardi ${ }^{2}$ iD \\ ${ }^{1}$ Department of Psychiatry, Weill-Cornell Medical College, New York, NY, USA. ${ }^{2}$ Laboratório Pânico e Respiração, Instituto de Psiquiatria, \\ Universidade Federal do Rio de Janeiro (UFRJ), Rio de Janeiro, RJ, Brazil.
}

Systems biology research can draw upon perspectives from all aspects of biological function to understand mechanisms, disease groups, and individual diseases. In this issue, Mufford et al. ${ }^{1}$ offer a marvelously coherent compendium of many diverse perspectives on anxiety disorders. The future bodes well for this type of overarching research, and existing studies illustrate the tantalizing prospects for ongoing investigations, crossmodality findings and, ultimately, improved clinical care. The article offers a roadmap of the many research paths to consider. ${ }^{1}$

Systemic investigation of anxiety disorders can be more difficult than for some other diagnostic groups. For one thing, research must rely on specific anxiety subtypes to make sense of data on biological mechanisms. Yet, there remain important theoretical and practical controversies about diagnosis. For example, official DSM diagnostic criteria have varied over the years. Does agoraphobia exist as a discrete entity, or is it always associated with panic anxiety? Does generalized anxiety disorder exist as a distinct entity, or do its common comorbidities with panic anxiety and social anxiety suggest that it is among the underlying and more specific causes of persistent anxiety? Is obsessive-compulsive disorder no longer an anxiety disorder, or does it have some biological similarities to other anxiety disorders? Are agitated depression and depression with anxiety caused in part by anxiety disorders, or are agitation and anxiety mere artifacts of certain types of depression?

Anxiety disorders are highly prevalent and economically burdensome psychiatric diagnoses, yet they are among the most commonly misdiagnosed and undertreated. ${ }^{2}$ The experience of anxiety has a universality that extends across time and culture. Only in the past few decades have researchers developed rigorous diagnostic criteria to improve clinical and basic research on the various anxiety disorders. ${ }^{1-3}$ The psychobiology of anxiety disorders is one of the most rewarding subjects of contemporary medical research. At least three central neurotransmitter systems (noradrenergic, serotonergic, and gamma-aminobutyric acid) are certainly affected by the various pharmacological compounds that provide therapeutic benefit. Some neurotransmitter systems have

Correspondence: Antonio E. Nardi, Rua Visconde de Pirajá, 407, sala 702, CEP 21941-901, Rio de Janeiro, RJ, Brazil.

E-mail: antonioenardi@gmail.com

Submitted Dec 20 2020, accepted Jan 04 2021, Epub Feb 122021. been found to underlie anxiety symptoms and disorders, and they are new challenges to investigators. ${ }^{1,2,4}$ The translation of neuroscience data has led to new insights into the etiology of anxiety disorders and supported the development of novel psychosocial and pharmacological treatments, including a better understanding of the interaction between genes and the environment. Animal studies have indicated that fear and anxiety-like states are mediated by structures that include the amygdala, hippocampus, prefrontal cortex, locus coeruleus, and periaqueductal gray matter. ${ }^{2,3}$

It may even be that there are discrete subtypes of recognized anxiety disorders whose biological properties vary. Respiratory panic anxiety and social performance anxiety come to mind, along with the five factoranalytically defined subtypes of obsessive-compulsive disorder. The systems biology of anxiety disorder may vary across the time course and acute phases of the illness. ${ }^{3}$ Consider acute panic attack vs. non-panic intervals vs. anticipatory anxiety. Some of the frequent comorbidities of anxiety disorders may make it more difficult to sort out biological findings on each one.

Certainly, current clinical diagnosis of anxiety disorders relies on a careful phenomenological interview. Anxiety rating scales may be useful for tracking symptoms and, occasionally, treatment response, but have no value for specific diagnosis. Even initial patient-reported severity ratings on such scales may change due to personality, culture or circumstances as much as to the actual biological severity of an anxiety disorder. There are many Stoics among us, as well as those who are more dramatic in reporting their distress. In panic anxiety, there are various challenge tests that appear to be specific for panic, with little response in panic-free controls. ${ }^{2}$ While sodium lactate infusions may be the best known induction method, carbon dioxide inhalation appears to be a simple, safe and inexpensive alternative. ${ }^{3}$ Fortunately, careful utilization of biological systems, clinical phenomenological-based interviews, biological data and some anxiety challenge tests, evolutionary theory, and pharmacological features can help us answer the remaining questions. ${ }^{1,3}$ Susceptibility to anxiety disorders may be determined early in life. Since the mechanisms of development are

How to cite this article: Kahn JP, Nardi AE. Anxiety disorders and systems biology research: a challenging brave new world. Braz J Psychiatry. 2021;43:351-352. http://dx.doi.org/10.1590/1516-44462020-1712 
under both genetic and environmental control, ${ }^{4}$ they can lead an organism to lifelong distress in response to threatening situations. According to recent data, ${ }^{4}$ genetic changes in some anxiety disorders, changes in the expression of the serotonin type $1 \mathrm{~B}$ receptor, and a decrease in hippocampal volume are associated with a predisposition to anxiety disorders and depression. ${ }^{4}$ It has been well documented in lab animals that environmental influences during early development, such as trauma from maternal separation or malnutrition, lead to anxiety disorders and more severe physiological changes. ${ }^{4}$ One explanation for this could be that the hippocampus is more susceptible to adverse influences during the early stages of brain development.

In addition to paying close attention to specific diagnostic entities, care must be taken that data show valid correlations, since the direction of causality can be uncertain. For example, transcriptome-wide association studies have found that downregulated amygdala and prefrontal expression of Ppm1f affects phosphatase stress response pathways. ${ }^{1}$ However, is phosphatase a contributor to stress, a compensatory response to stress, or are both it and stress results of a common underlying cause? Similar questions arise about the association found in genome-wide association studies between panic disorder and TMEM132D variants, which are also associated with phosphatase activity. The findings of epigenome-wide association research regarding hypomethylation in panic-associated regions are fascinating and consistent with data which suggest that early childhood has epigenetic effects on panic. ${ }^{1}$ But here too, the directions of causality are crucial. ${ }^{1-4}$

Some simple and science-based considerations should also be highlighted, since it is time to return to the evidence about benzodiazepines and conceptual rigor in interpreting it. ${ }^{5}$ Ample evidence indicates that benzodiazepines are highly effective in the treatment of anxiety disorders; those who deny their efficacy and safety should listen more carefully to their patients. Accepting unsupported criticism about benzodiazepines will perpetuate stigma against benzodiazepines, the clinicians who prescribe them and the patients who take them. Clinicians should invite colleagues to engage in evidence-based reappraisal of the benefits and risks of these medications and abandon aspects of conventional wisdom that do not stand up to scrutiny. ${ }^{5}$

In untangling these issues, one cue might be taken from trans-diagnostic studies of personality and psychotic disorders. ${ }^{4}$ Factor analysis of a many personality measures yielded the five-factor model of personality, while factor analysis of psychosis symptoms has also yielded five psychosis subtypes. When further data on systems biology are available, a factor analysis that also includes clinical information might point to specific anxiety subtypes and their characteristics. As Mufford et al. ${ }^{1}$ has pointed out, we now have a proof of concept with increasingly precise and useful findings. Amazing new understanding about etiology, valid clinical diagnosis, and better treatments may be around the corner.

\section{Disclosure}

The authors report no conflicts of interest.

\section{References}

1 Mufford MS, van der Meer D, Andreassen OA, Ramesar R, Stein DJ, Dalvie S. A review of systems biology research of anxiety disorders. Braz J Psychiatry. 2021;43:414-23.

2 Nardi AE, Fontenelle LF, Crippa JA. New trends in anxiety disorders. Braz J Psychiatry. 2012;34 Suppl 1:S5-6.

3 da Luz AC, Dias GP, Bevilaqua MC, Cocks G, Gardino PF, Thuret S, et al. Translational findings on brain-derived neurotrophic factor and anxiety: contributions from basic research to clinical practice. Neuropsychobiology. 2013;68:129-38.

4 Lopes FL, Zhu K, Purves KL, Song C, Ahn K, Hou L, et al. Polygenic risk for anxiety influences anxiety comorbidity and suicidal behavior in bipolar disorder. Transl Psychiatry. 2020;10:298.

5 Silberman E, Balon R, Starcevic V, Shader R, Cosci F, Fava GA, et al. Benzodiazepines: it's time to return to the evidence. $\mathrm{Br} \mathrm{J}$ Psychiatry. 2021;218:125-7. 